\title{
Priapismo: comorbilidades, tratamiento, complicaciones y función sexual de los pacientes
}

Carrillo-Córdova LD, Acevedo-García C, Rivera-Astorga H, Garduño-Arteaga LM

\section{Resumen}

ANTECEDENTES: el priapismo es una alteración poco frecuente, con principal afectación de los cuerpos cavernosos, que resulta en erección persistente del pene, acompañada o no por el coito o estimulación sexual, con duración de 6 horas o más.

OBJETIVO: estimar la prevalencia de comorbilidades, tratamiento y complicaciones asociadas en pacientes con priapismo.

MATERIALES Y MÉTODOS: estudio retrospectivo, del que se recabó la información de los expedientes clínicos de pacientes con diagnóstico de priapismo. Se evaluaron las comorbilidades, tratamiento, complicaciones y resultados asociados con la enfermedad. El diagnóstico de priapismo se estableció con base en la clínica y se confirmó mediante la gasometría de cuerpos cavernosos; el tratamiento de los pacientes se estableció según las guías de la American Urological Association y la European Association of Urology. La decisión del tipo de derivación caverno-esponjosa se seleccionó con base en el criterio del cirujano tratante. Las complicaciones tempranas fueron descritas según la clasificación de Clavien-Dindo. La recuperación de la función eréctil se evaluó mediante llamadas telefónicas.

RESULTADOS: se registraron 14 pacientes (13 con priapismo isquémico de bajo flujo confirmado mediante gasometría $[\mathrm{pH}<7.23$ ] y 1 con priapismo de alto flujo); la comorbilidad más frecuentemente asociada fue la enfermedad mental, en la que se incluyó el abuso de sustancias. Las complicaciones aguda y crónica más comunes fueron cavernositis y disfunción eréctil, respectivamente. De los pacientes con priapismo isquémico, solo 1 tuvo curación con tratamiento conservador (AINES) y los 12 restantes con irrigación intracavernosa.

CONCLUSIONES: existe gran prevalencia de enfermedades psiquiátricas en pacientes con priapismo. La tasa de disfunción eréctil es superior a la reportada en otras series. Con este estudio esperamos mejorar el conocimiento del priapismo en nuestra población.

PALABRAS CLAVE: priapismo, comorbilidades, complicaciones, disfunción eréctil.
Departamento de Urología, Hospital General de México Dr. Eduardo Liceaga, Ciudad de México.

Recibido: diciembre 2016

Aceptado: septiembre 2017

Correspondencia

Luis Daniel Carrillo Córdova

carrillocor@gmail.com

Este artículo debe citarse como

Carrillo-Córdova LD, Acevedo-García C, Rivera-Astorga H, Garduño-Arteaga LM. Priapismo: comorbilidades, tratamiento, complicaciones y función sexual de los pacientes. Rev Mex Urol. 2017 sep-oct;77(5):383-388. DOI: https://doi.org/10.24245/revmexurol.v77i5.1120 


\section{Priapism: Comorbidities, treatment, complications, and sexual function}

Carrillo-Córdova LD, Acevedo-García C, Rivera-Astorga H, Garduño-Arteaga LM

\begin{abstract}
BACKGROUND: Priapism is an uncommon alteration that mainly affects the corpora cavernosa, resulting in a persistently erect penis with or without sexual stimulation, lasting for 6 hours or more.
\end{abstract}

OBJECTIVE: To estimate the prevalence of comorbidities, treatment, complications, and results associated with patients with priapism.

MATERIALS AND METHODS: A retrospective study employing case record information on patients diagnosed with priapism was conducted. Comorbidities, treatment, complications and results associated with the disease were evaluated. Diagnosis was clinical and confirmed through blood gasometry of the corpora cavernosa. Treatment followed the American Urological Association and the European Association of Urology guidelines. The decision of which type of corporo-spongiosal shunt to use was based on the treating surgeon's criterion. Early complications were described according to the Clavien-Dindo classification. Erectile function recovery was evaluated through a telephone interview.

RESULTS: Fourteen patients were enrolled in the study (13 with lowflow priapism confirmed through gasometry $[\mathrm{pH}<7.23]$ and 1 with high-flow priapism). The most frequently associated comorbidity was mental illness, which included substance abuse. Cavernositis was the most common acute complication and erectile dysfunction was the most common chronic complication. Of the patients with ischemic priapism, only 1 was cured with conservative treatment (NSAID) and the remaining 12 with intracavernous irrigation.

CONCLUSIONS: Psychiatric illnesses were highly prevalent in the patients with priapism and the erectile dysfunction rate was higher than that reported in other case series. Our study results can improve knowledge of priapism in the Mexican population.

KEYWORDS: Priapism; Comorbidities; Complications; Erectile dysfunction
Departamento de Urología, Hospital General de México Dr. Eduardo Liceaga, Ciudad de México.

Correspondence Luis Daniel Carrillo Córdova carrillocor@gmail.com

\section{ANTECEDENTES}

El priapismo es una afectación de los cuerpos cavernosos, que resulta en erección persistente del pene, acompañada o no por el coito o estimulación sexual, con duración de 6 horas o más. La erección puede originarse en su porción dorsal, con la permanencia del pene ventral y el 
glande en estado flácido. ${ }^{1}$ Esta alteración es una urgencia urológica y puede deberse a distintos trastornos. Las guías de la American Urological Association, European Association of Urology y la British Association of Urological Surgeons se encargan de establecer los protocolos de tratamiento en estos pacientes. ${ }^{2-4}$

Un estudio de cohorte retrospectivo encontró que la incidencia de priapismo es de 1.5 en la población general y de 2.9 en hombres mayores de 40 años de edad, por cada 100,000 personas-años. ${ }^{5}$ Los pacientes que reciben inyecciones intracavernosas de prostaglandinas $E_{1}$ para el tratamiento de la disfunción eréctil han reportado una incidencia de priapismo de $1 \%$ y para papaverina de $17 \% .{ }^{6}$ En niños con anemia drepanocítica, la incidencia varía de 6 a 27\%. ${ }^{7,8}$ En adultos, la incidencia aumenta, incluso, de 42 a $89 \% .{ }^{9,10}$

Entre los factores asociados con el priapismo se encuentran los trastornos hematológicos y algunos medicamentos; sin embargo, es importante reconocer otros factores de riesgo para aumentar el conocimiento y prevenir esta rara alteración.

El objetivo de este estudio fue estimar la prevalencia de comorbilidades, tratamiento, y complicaciones relacionadas en pacientes con priapismo.

\section{MATERIALES Y MÉTODOS}

Estudio retrospectivo, del que se recabó la información de los expedientes clínicos, o que coincidieran con el código ICD-9 (607.3), de pacientes con diagnóstico de priapismo, quienes acudieron al Departamento de Urología del Hospital General de México Dr. Eduardo Liceaga, entre los años 2010 y 2016. Se revisaron y capturaron los datos contenidos en los expedientes clínicos, con especial interés en las comorbilidades, tratamiento, complicaciones y resultados. El diagnóstico de priapismo se estableció con base en la clínica y se confirmó mediante la gasometría de cuerpos cavernosos; el tratamiento se estableció según las guías de la American Urological Association y la European Association of Urology.

La decisión del tipo de derivación cavernoesponjosa se seleccionó con base en el criterio del cirujano tratante, principalmente las variantes: Al-Ghorab (en 6 pacientes), Winter (en 12 pacientes) y Ebbehoj (en 3 pacientes), incluso se evaluó si algunos pacientes requirieron más de una técnica, iniciando siempre con derivación distal, seguida de derivación proximal.

Las complicaciones tempranas fueron descritas según la clasificación de Clavien-Dindo. ${ }^{11}$ La recuperación de la función eréctil se evaluó mediante Ilamadas telefónicas, en las que se dicotomizó en la variable; la pregunta efectuada a los pacientes fue ¿es usted capaz de tener erecciones lo suficientemente buenas, que le permitan tener relaciones sexuales? Sí o no.

\section{RESULTADOS}

Se identificaron 14 pacientes con diagnóstico de priapismo, de acuerdo con el ICD-9 (607.3): 13 con priapismo isquémico de bajo flujo confirmado mediante gasometría ( $\mathrm{pH}<7.23)$, de los que $28 \%$ tuvieron recurrencia ( 1 a 4 veces), y 1 con priapismo de alto flujo, en quien se realizó ultrasonido Doppler, arteriografía pudenda y embolización, como parte del diagnóstico y tratamiento (Cuadro 1).

Cuadro 1. Tipo de priapismo

\begin{tabular}{l|c|c|c|} 
& $(\mathbf{n})$ & Edad (años) & Duración (horas) \\
\hline Isquémico & 13 & $35(26-47)$ & $70(24-88)$ \\
\hline Arterial & 1 & 25 & 144 \\
\hline
\end{tabular}


En los 13 pacientes con priapismo isquémico se efectuó ultrasonido de pene $(n=12)$, con promedio de índice de resistencia de la arteria dorsal del pene de 0.65 (0.57-0.77) y velocidad de flujo de $9.3 \mathrm{~cm} / \mathrm{seg}$ (7.5-13.2). Los hallazgos gasométricos de pacientes con priapismo isquémico fueron: $\mathrm{pH} 7.1$ (6.8-7.2), $\mathrm{pO}_{2} 35.6$ (7-71.2), $\mathrm{pCO}_{2} 61.4$ (10-120); hemoglobina 14.6 (8.1-21), hematocrito 45.1 (27-67) y lactato 10.4 (2-15); y de quienes tuvieron priapismo arterial: $\mathrm{pH} 7.4$, $\mathrm{pO}_{2} 59$, $\mathrm{pCO}_{2}$ 59; hemoglobina 12 , hematocrito 36 y lactato 1.9 (Cuadro 2).

Las comorbilidades de nuestra población se describen en el Cuadro 3. La principal alteración en los pacientes con priapismo isquémico fue la enfermedad mental (considerando el abuso de sustancias, según el DSM_IV que lo clasifica

Cuadro 2. Variables gasométricas (priapismo isquémico)

\begin{tabular}{|l|c|c|c|c|c|c|} 
& $\mathrm{pH}$ & $\mathrm{p0}_{2}$ & $\mathrm{Hb}$ & $\mathrm{Htco}$ & $\mathrm{Lac}$ & $\mathrm{pCO}_{2}$ \\
\hline Media & 7.1 & 35.6 & 14.6 & 45.2 & 10.4 & 61.4 \\
\hline DE & 0.2 & 27.9 & 4.6 & 14.2 & 4.3 & 37.8 \\
\hline Mínimo & 6.8 & 7 & 8.1 & 27 & 2 & 10 \\
\hline Máximo & 7.2 & 71.3 & 210 & 67 & 15 & 120.6 \\
\hline
\end{tabular}

Hb: hemoglobina; Htco: hematocrito; Lac: lactato.

Cuadro 3. Comorbilidades en pacientes con priapismo

\begin{tabular}{|l|c|}
\hline Comorbilidades & (n) \\
\hline Enfermedad mental & 10 \\
\hline $\begin{array}{l}\text { Abuso de sustancias } \\
\text { (Cocaína, mariguana, alcohol) }\end{array}$ & 6 \\
\hline $\begin{array}{l}\text { Esquizofrenia } \\
\text { (Risperidona) }\end{array}$ & 3 \\
\hline Trastorno depresivo & 1 \\
\hline Tabaquismo & 7 \\
\hline Consumo de sildenafilo & 1 \\
\hline Sin comorbilidades & 3 \\
\hline Leucemia o linfoma & 2 \\
\hline Hipertensión & 4 \\
\hline
\end{tabular}

Algunos pacientes tuvieron más de una comorbilidad. como Trastornos relacionados con sustancias); este diagnóstico se estableció en $76 \%$ de los casos, y de éstos el más frecuente fue el abuso de sustancias $(60 \%)$. Otros trastornos encontrados fueron: esquizofrenia y depresión.

Las sustancias con mayor abuso fueron: cocaína $(n=4)$, marihuana $(n=1)$ y alcohol $(n=1)$, y los medicamento: risperidona $(n=3)$, sertralina $(n=1)$ y citalopram $(n=1)$. Dos pacientes tenían diagnóstico de leucemia, 7 refirieron consumo de tabaco, 4 hipertensión y 1 caso con priapismo asociado con sildenafilo. Solo 3 pacientes no registraron ningún tipo de comorbilidad. Se encontró 1 paciente con priapismo de alto flujo asociado con fístula arteriovenosa, con antecedente de traumatismo pélvico-perineal durante la infancia.

De los pacientes con priapismo isquémico, solo 1 tuvo curación con tratamiento conservador (AINES); en los 12 restantes el tratamiento inicial consistió en irrigación intracavernosa (4 pacientes con curación), seguido de cortocircuito distal (con resolución de tres pacientes) $y$, finalmente, un segundo cortocircuito distal o proximal $(n=1)$, con curación de los 5 casos restantes (Cuadro 4). El 80\% de las derivaciones distales se realizó en la sala de urgencias y $20 \%$ en el quirófano.

Cuadro 4. Tratamiento del priapismo

\begin{tabular}{|l|l|}
\hline Tratamiento & (n) \\
\hline Observación (sin tratamiento) & 1 \\
\hline Embolización arterial & 1 \\
\hline Irrigación & 4 \\
\hline Irrigación seguida de cortocircuito & 3 \\
\hline Segundo cortocircuito & 5 \\
\hline Procedimientos por paciente & \\
\hline 1 & 6 \\
\hline 2 & 3 \\
\hline 3 & 5 \\
\hline 4 & 0 \\
\hline
\end{tabular}


Entre las complicaciones tempranas y tardías relacionadas con el tratamiento se encontraron 4 pacientes con infección de herida quirúrgica y hematoma perineal, y disfunción eréctil (64\%), respectivamente (Cuadro 5).

\section{DISCUSIÓN}

Este estudio retrospectivo es el más grande reportado en la población latinoamericana. No se encontró ningún caso de anemia drepanocítica, como lo señalan algunas series norteamericanas y europeas; sin embargo, se registró elevada prevalencia de trastornos psiquiátricos asociados con el abuso de sustancias, similar a lo reportado por Gottsch y su grupo. ${ }^{12}$ Estos autores reportaron una prevalencia de $56 \%$ de abuso de sustancias, además de lesión medular. El 21\% (n =3) de nuestros pacientes no manifestó ninguna comorbilidad, dato similar (18\%) al obtenido por otros investigadores. ${ }^{12-14}$

En una serie nigeriana de 40 pacientes, la alteración más común relacionada con priapismo fue la anemia de células falciformes (82.5\%), quizá por la gran prevalencia de la enfermedad en el continente africano. El estudio de Gottsch y su equipo de trabajo reportó 2 pacientes con esta alteración. ${ }^{14-15}$ Una serie de 207 pacientes encontró una diferencia significativamente más alta de priapismo en pacientes que consumían alcohol o medicamentos psicotrópicos versus controles sanos, y concluyó que el priapismo puede tener un origen multifactorial. ${ }^{16}$
La relación entre salud mental y función eréctil aún se desconoce; sin embargo, algunos estudios reportan una importante asociación entre enfermedades psiquiátricas y priapismo, además del tratamiento con medicamentos antipsicóti$\cos _{,}{ }_{13-16}$ cuyos mecanismos moleculares pueden deberse al bloqueo de los receptores alfa- 1 adrenérgicos..$^{15,17}$ Incluso se ha propuesto que los pacientes con priapismo que consumen cocaína padecen desregulación autonómica. ${ }^{18-22}$

La complicación temprana más frecuente en este estudio fue la infección de herida quirúrgica, que coincide con las primeras series reportadas, ${ }^{23}$ y la tardía, la disfunción eréctil, que se ha relacionado con priapismo en más de $35 \%$ de los pacientes. ${ }^{11-17}$ En nuestra serie encontramos un porcentaje elevado de pacientes con disfunción eréctil $(68 \%)$, superior al reportado en la bibliografía y similar a la investigación de Ahmed y su grupo $(60 \%),{ }^{14}$ que puede deberse al tiempo prolongado de evolución de la enfermedad en los pacientes, incluso antes de acudir a nuestra institución (promedio de 70 horas). Además, otras series han reportado fibrilación auricular, pero en nuestro estudio no encontramos ningún caso relacionado. ${ }^{12}$

Hace poco se publicó una serie de 12 pacientes con diagnóstico de priapismo en la población mexicana: 10 de origen isquémico, 1 arterial y 1 recurrente; sin embargo, el estudio no señala el origen del priapismo, las complicaciones ni la función sexual después de establecer el tratamiento. ${ }^{23}$

Cuadro 5. Complicaciones de pacientes con priapismo

\begin{tabular}{l|l}
$\begin{array}{l}\text { Procedimiento } \\
\text { Cortocircuito distal }\end{array}$ & (n) Complicación (Clavien-Dindo) y tratamiento \\
$\begin{array}{l}\text { Infección de herida (3) } \rightarrow \text { Antibiótico (Clavien-Dindo II) } \\
\text { Cortocircuito proximal }\end{array}$ & $\begin{array}{l}\text { Hematoma perineal }(1) \rightarrow \text { Evacuación (Clavien-Dindo IIIB) } \\
\text { Infección de herida perineal }(1) \rightarrow \text { Antibiótico y aseo de herida (Clavien-Dindo IIIB) }\end{array}$ \\
\hline Potencia Sexual & (n) \\
\hline Sí & 5 \\
No & 9
\end{tabular}




\section{CONCLUSIONES}

Encontramos gran prevalencia de enfermedades psiquiátricas en pacientes con priapismo y la tasa de disfunción eréctil que reportamos en este trabajo es superior a la de otras series. Con este estudio esperamos mejorar el conocimiento del priapismo en nuestra población.

Entre las limitaciones del estudio encontramos una población pequeña $(n=14)$; por tratarse de una cohorte retrospectiva no es posible conocer si todas las complicaciones fueron capturadas en el expediente; no se utilizó un cuestionario validado para evaluar la función eréctil, pues por tratarse de un dato autoinformado por el paciente mediante una Ilamada telefónica, fue imposible verificarlo.

\section{Conflicto de interés}

Los autores declaran no tener conflicto de interés.

\section{REFERENCIAS}

1. Montague DK, Jarow J, Broderick GA, et al. American Urological Association Guideline On The Management Of Priapism. J Urol. 2003;170(4):1318-1324.

2. Muneer A, Ralph D. Guideline of Guidelines Priapism. BJU Int. 2016;119(2);204-8.

3. Salonia A, Eardley I, Giuliano F, Hatzichristou D, Moncada I, Vardi Y, Wespes E, Hatzimouratidis K. European Association of Urology_guidelines on_priapism. European Association of Urology. Eur Urol;2014;65(2):480-9.

4. Eland IA, van der Lei J, Stricker BH, Sturkenboom MJ. Incidence of priapism in the general population. Urology 2001;57:970-2.

5. Linet OI, Ogrinc FG. Efficacy and safety of intracavernosal alprostadil in men with erectile dysfunction. The Alprostadil Study Group. N Engl J Med. 1996;334:873-7.

6. Tarry WF, Duckett JW, Synder HM. Urological complications of sickle cell disease in a pediatric population. J Urol. 1987;138:592-4-7

7. Ewalt D, Cavender J, Buchanan G, Rogers Z. Leuprolide therapy prevents recurrent priapism in teenage boys with SCA. Paediatrics. 1996; 88:643.
8. Mantadakis E, Cavender JD, Rogers ZR, Ewalt DH, Buchanan GR. Prevalence of priapism in children and adolescents with sickle cell anemia. J Pediatr Hematol Oncol. 1999;21:518-22.

9. Burnett AL. Pathophysiology of priapism: dysregulatory erection physiology thesis. 2003;170:26-34.

10. Dindo D, Demartines N, Clavien PA, Classification of surgical complications: a new proposal with evaluation in a cohort of 6336 patients and results of a survey. Ann Surg. 2004;240(2):205-213.

11. Gottsch HP, Berger RE, Yang CC. Priapism: comorbid factors and treatment outcomes in a contemporary series. Adv Urol. 2012;2012:672624. doi: 10.1155/2012/672624. Epub 2012 Jul 3.

12. Fu E, Kovach JG, Dubin WR. priapism associated with antipsychotic medication use: case report. J Clin Psychopharmacol 2017;37(4):477-8R.

13. Fiorelli L, Manfrey SJ, Belkoff LH, Finkelstein LH. Priapism associated with intranasal cocaine abuse. J Urol. 1990;143(3):584-585.

14. Ahmed M, Augustine B, Matthew M, Awaisu M, Sudi A, Hamza KB, et al. Prognostic factors and outcome of management of ischemic priapism in Zaria, Nigeria. Niger J Surg. 2017;23(1):15-9.

15. Munarriz R, Hwang J, Goldstein I, Traish AM, Kim NN Cocaine and ephedrine-induced priapism: case reports and investigation of potential adrenergic mechanisms. Urology-2003;62(1):187-192.

16. Kulmala $R$, Lehtonen $T$, Nieminen $P$, Tammela T. Aetiology of priapism in 207 patients. Eur Urol. 1995;28(3):241-245.

17. Andersohn F, Schmedt N, Weinmann S, Willich SN, Garbe E. Priapism associated with antipsychotics: role of $\alpha 1$ adrenoceptor affinity. J Clin Psychopharmacol. 2010;30(1);68-71.

18. Bar KJ, Boettger MK, Schulz S, et al. Reduced cardio-respiratory coupling in acute alcohol withdrawal. Drug Alcohol Depend. 2008;98(3):210--217.

19. Vongpatanasin W, Mansour Y, Chavoshan B, Arbique D. Cocaine stimulates the human cardiovascular system via a central mechanism of action. Circulation.1999;100(5):497-502.

20. Henry BL, Minassian A, Paulus MP, Geyer MG, Perry W. Heart rate variability in bipolar mania and schizophrenia. J Psychiatric Res. 2010;44(3):168-176.

21. Carney RM, Blumenthal JA, Stein PK, et al. Depression, heart rate variability, and acute myocardial infarction. Circulation. 2001;104(17):2024-2028.

22. Hinman F. Priapism: report of cases and a clinical study of the literature with reference to its pathogenesis and surgical treatment. Ann Surg. 1914;60:689-716.

23. Torres-Ochoa DE, Delgado-Guerrero F, Torres-Aguilar J, Lugo-García JA. Etiología y manejo de priapismo en el Hospital Juárez de México. Bol Coleg Mex Urol 2016;31(3):87-89. 